\title{
Satellites are the main drivers of environmental effects at least to $\mathrm{z}=0.7$
}

\author{
Katarina Kovač ${ }^{1}$ and the zCOSMOS $\dagger$ team \\ ${ }^{1}$ Institute for Astronomy, ETH Zurich, Zurich 8093, Switzerland \\ email: kovac@phys.astro.ch
}

\begin{abstract}
We study the role of environment in the evolution of galaxies up to $\mathrm{z}=0.7$ using the final zCOSMOS-bright data set. We use the colour as a proxy for the quenched population, and measure the dependence of the red fraction of galaxies on stellar mass and two environmental indicators: the local overdensity of galaxies $\delta$ and a demarcation of galaxies to centrals and satellites. The analysis is carried out by quantifying the role of different quenching processes. We find that the measured dependence of the red fraction of galaxies on stellar mass and environment can be well described by two quenching processes: one related only to stellar mass (mass quenching) and the other related to the local environment (environment quenching). Within the errors, these processes are independent of each other, and consistent with the $z \sim 0$ measurement. Moreover, the red fraction of centrals $f_{r, c e n}$ (both singleton centrals and centrals in the groups) does not show any trend with $\delta$ and more than $95 \%$ of $f_{r, c e n}$ is consistent with being produced through the mass quenching alone. The satellite galaxies are redder than the centrals at the same stellar mass and $\delta$, requiring additional environment quenching. Given the observed fractional distribution of satellites at different overdensities, the normalized excess in the red fraction of satellites with respect to the red fraction of centrals is consistent with a scenario in which the satellites account for most of the $\delta$-dependences observed in the overall population of galaxies covering $0.1<z<0.7$.
\end{abstract}

Keywords. galaxies: evolution, cosmology: large-scale structure of universe

\section{Introduction}

A correlation between various galaxy properties and their environment has been measured up to redshifts of about $z=1-1.5$ (e.g. Dressler 1980; Cooper et al. 2010; Kovač et al. 2014). Galaxies which reside in the regions of higher density are more massive, redder, they are forming less stars, and morphologically they are of an earlier class than their counterparts in the less dense environments (e.g. Kauffmann et al. 2004; Cucciati et al. 2010; Kovač et al. 2010a).

There is a range of physical processes suggested to exist only in dense environments (see e.g. Boselli \& Gavazzi 2006), where the majority if not all of the suggested mechanisms are related to the transformation of galaxies after they become satellites. With the recent advances in the spectroscopic surveys, there is growing observational evidence that satellite galaxies are indeed affected by some additional environment specific processes. At the same stellar mass, the fraction of quenched satellites is systematically above those of the centrals, consistent with a scenario in which $40-50 \%$ of star-forming galaxies transition to quenched galaxies after they infall into a more massive halo (van den Bosch et al. 2008; Peng et al. 2012). This fraction stays remarkably constant with stellar mass and redshift up to $z=0.8$ (Knobel et al. 2013).

$\dagger$ Based on observations undertaken at the European Southern Observatory (ESO) Very Large Telescope (VLT) under Large Program 175.A-0839. 
The fraction of quenched satellites also increases with the environmental overdensity, and locally, satellite quenching can account for most of the observed environmental dependence in the quenched fraction of all galaxies (Peng et al. 2012). We showed in Kovač et al. (2014) that to a good degree this holds to $z=0.7$ and in this proceeding, we will highlight the key points from that work. Our recent and ongoing more detailed analysis of the observational data shows that the central/satellite paradigm is more complicated: e.g. for massive centrals in the rich groups at $z \sim 0$ the quenched fractions of centrals and satellites are the same (Knobel et al. 2014) and the fraction of quenched satellites is correlated with the properties of their central over the whole $0<z<0.7$ interval (i.e. galactic conformity, Knobel et al. 2014, Kovač et al. in prep).

\section{Data sample}

Our analysis is based on the final zCOSMOS-bright data set of $\sim 17,000$ galaxies with reliable redshift at $z<1.2$ (Lilly et al. 2007, 2009). This sample is limited by a magnitude $i_{A C S}=22.5$ and it is confined to $1.4 \mathrm{deg}^{2}$ within the COSMOS field (Scoville et al. 2007). The properties of galaxies (such as luminosities and stellar masses) are based on the the spectral energy distribution (SED) fitting utilising a subset of the 30-band COSMOS photometry (e.g. Capak et al. 2007; Sanders et al. 2007).

We use two indicators of environment: local overdensity of galaxies and the identification of a galaxy as a central or a satellite. The apertures for the overdensity reconstruction are obtained by measuring distances to the fifth nearest neighbour in the $M_{B}<-19.3-z$ sample of galaxies which includes both galaxies with the reliable redshifts and the ZADE-modified photometric probability distribution functions (see Kovač et al. 2010b). The central/satellite identification of galaxies is based on the group catalogue of Knobel et al. (2012). By definition, the most massive galaxy in a group is termed central, and other galaxies in a given group are marked as satellites. We use the mocks (Henriques et al. 2012), modified to match the zCOSMOS selections, to quantify the imperfect separation of galaxies to centrals and satellites and use these corrections to obtain the statistically corrected, true quantities.

The samples which we use in the subsequent analysis contain 2340, 1730, and 610 all, central, and satellite galaxies above the adopted mass limit in $0.1<z<0.4$. In the higher redshift bin $0.4<z<0.7$, these numbers are 2448, 2062, and 386 respectively. The mass limits are $\log \left(M_{*} / M_{\odot}\right)=9.82$ and 10.29 in the lower and higher redshift bins.

\section{Colour-density relation for the overall sample}

We start by quantifying the relation between the red fraction $f_{\text {red }}$ of all zCOSMOS galaxies above the mass completeness limit and their local overdensity and stellar mass. The measured red fractions as a function of overdensity are shown in Fig. 1 for a range of independent stellar mass bins. Even though some of the measured points are discrepant, there is an obvious trend that at a given stellar mass the red fraction of galaxies increases with the local overdensity. Also, at a given value of overdensity, the red fraction increases with the stellar mass. These relations agree (at least qualitatively) with the previously published similar analyses based on the smaller 10k zCOSMOS data set (Cucciati et al. 2010; Tasca et al. 2009) and a broad range of studies from other surveys up to $z=1.5$ (e.g. Baldry et al. 2006; Peng et al. 2010; Cooper et al. 2010).

It was shown by Baldry et al. (2006) that at $z \sim 0$ (see also Peng et al. 2010 for $0.3<z<0.6$ ) the dependence of the red fraction on both stellar mass and overdensity can be well quantified by the following function: 

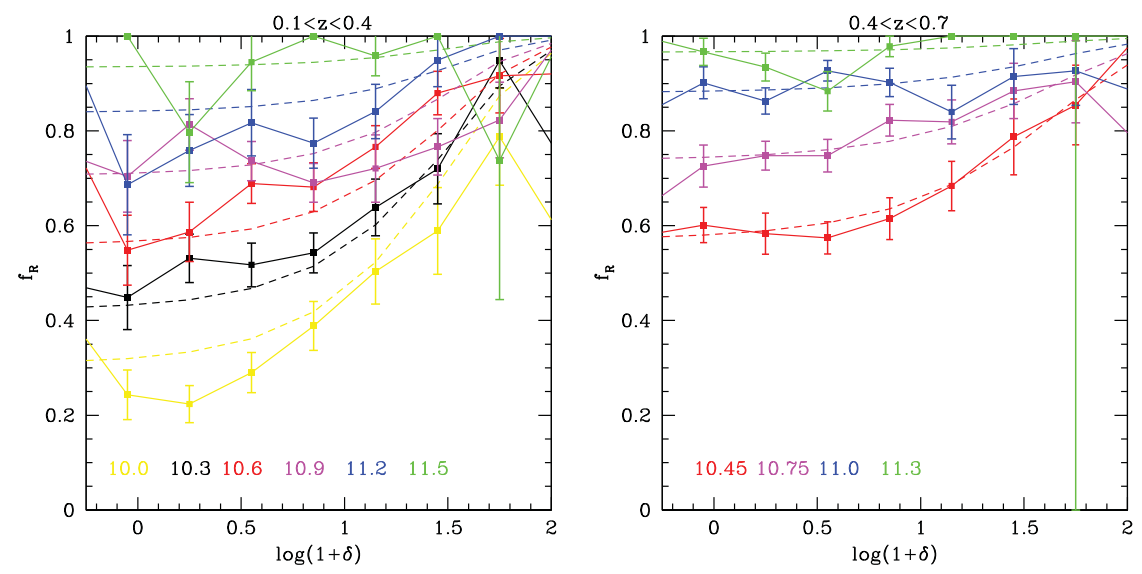

Figure 1. Red fraction of all zCOSMOS galaxies as a function of overdensity in different bins of stellar mass. The left- and right-hand panels correspond to $0.1<z<0.4$ and $0.4<z<0.7$, respectively. The symbols, connected by solid lines, mark the measured fractions. Going from the bottom to the top, the stellar mass for each curve increases, and the median mass in an increasing order is given at the bottom of each of the panels. The dashed lines correspond to the best-fit model given by equation 3.1. The error bars correspond to half of the $16-84 \%$ interval from 100 bootstrapped samples. The figure is taken from Kovač et al. (2014, Fig. 3).

$$
f_{\text {red }}\left(\delta, M_{*}\right)=1-\exp \left(-\left(\delta / p_{1}\right)^{p 2}-\left(M_{*} / p_{3}\right)^{p 4}\right)
$$

where the $p 1-p 4$ parameters are simply the fitting parameters. We explore here if this function can still describe our final zCOSMOS data set. The red fraction from the best-fit model given by Eq. 3.1 is also shown in Fig. 1 (dashed lines). We conclude that the proposed model provides a good description of the zCOSMOS data, as most of the measured and fitted values agree within the $2 \sigma$ interval. Our additional error analysis does not indicate any systematic effect neither with the mass nor the density.

It is important to stress that one of the properties of the function given by equation 3.1 is that it is separable in its dependency on the mass and environment and it can be rewritten as:

$$
f_{\text {red }}\left(\delta, M_{*}\right)=\epsilon_{\rho}\left(\delta, \delta_{0}\right)+\epsilon_{m}\left(M_{*}, M_{* 0}\right)-\epsilon_{\rho}\left(\delta, \delta_{0}\right) \epsilon_{m}\left(M_{*}, M_{* 0}\right)
$$

where $\epsilon_{\rho}\left(\delta, \delta_{0}\right)=1-\exp \left(-\left(\delta / p_{1}\right)^{p 2}\right)$ and $\epsilon_{m}\left(M_{*}, M_{* 0}\right)=1-\exp \left(-\left(M_{*} / p_{3}\right)^{p 4}\right)(\mathrm{Peng}$ et al. 2010). Given the definition above, the $\epsilon_{\rho}$ function depends only on environment and the $\epsilon_{m}$ functions depends only on mass. Following Peng et al. (2010) we will refer to these two functions as the relative environment and mass quenching efficiencies, respectively. This therefore means that the red fraction of galaxies can be considered to be build through two different quenching processes, one of them dependent only on local density, and the other one dependent only on stellar mass or a closely related property. It is also plausible that the $5-15 \%$ difference between the measurement and the best-fit model reflects some quenching processes not related to mass or environment or depending on both of them simultaneously which are not included in this simple $f_{\text {red }}$ model.

A comparison of quenching efficiencies obtained from the zCOSMOS data in two redshift bins and from the $z \sim 0$ data (Peng et al. 2010) shows that, within the errors, there is no significant redshift evolution in the $\epsilon_{\rho}$ and $\epsilon_{m}$ functions. This however does not correspond to the static situation in the overall transition of galaxy population from blue 
to red. This simply means that the physical processes which are responsible for the mass and enviornment quenching act in the same way on galaxies of the same stellar mass and which reside in the quantitatively same environment at different epochs.

Moreover, the relative roles of the environment and mass quenching are expected to change with cosmic time (Fig. 15 in Peng et al. 2010), such that the role of environment increases with decreasing stellar mass and decreasing redshift. Qualitatively, this is consistent with the idea that the environment quenching affects the galaxies infalling into the larger haloes, building the large scale structure as cosmic time passes.

\section{Red fraction of centrals and satellites}

In this section we investigate the role of satellites in producing the observed colourdensity relation in the overall population of zCOSMOS galaxies. Given the relatively small number of satellites in our mass complete samples, we proceed by measuring the red fractions of centrals $f_{r, c e n}$ and satellites $f_{r, s a t}$ in a range of overdensity bins in the carefully constructed mass-matched samples. Centrals and satellites are divided in four quartiles of overdensity (defined by centrals and satellites, respectively, and for each redshift bin) and all samples are matched to the mass distribution of satellites in the reference sample. This was chosen to be the 37.5-62.5\% interval in the sorted satellite overdensitites. The median stellar mass is $\log \left(M_{*} / M_{\odot}\right)=10.40$ and 10.59 in the lower and higher redshift bin, respectively.

The resulting $f_{r, c e n}$, statistically corrected for the impurities in the central/satellite demarcation, are shown in the left-hand panels in Fig. 2 in the four $\delta$-quartiles. Broadly, we do not detect any strong trend between the red fraction of central galaxies and the overdensity in either of the redshift bins. The average red fractions of centrals (dotted lines in the left-hand panels in Fig. 2) are a few percent higher than or consistent with the red fractions expected from the mass quenching alone, estimated to be 0.48 and 0.66 at the median stellar mass in the reference samples in the lower and higher redshift bins, respectively (dashed lines in the left-hand panels in Fig. 2). As the environment quenching accounts for producing less than $5 \%$ of red centrals, we adopt in what follows that $f_{r, c e n}$ is independent of environment at all overdensities and in both redshift intervals. We take the average red fraction to be a proxy for $f_{r, c e n}$. As we do not have a sufficiently large sample of centrals at the highest overdensities probed by the satellites, this statement remains to be tested by future observations.

The purity corrected red fraction of satellites are shown as squares in the left-hand panels in Fig. 2. It is clear that the red fractions of satellites are systematically above the red fractions of centrals at all overdensities over $0.1<z<0.7$, requiring some additional quenching channel on top of the mass quenching. We quantify the excess of red satellites with respect to the centrals at a given overdensity $\delta$ and mass $M_{*}$ by defining the satellite quenching efficiency $\epsilon_{\text {sat }}\left(\delta, M_{*}\right)$ as

$$
\epsilon_{\text {sat }}\left(\delta, M_{*}\right)=\frac{f_{r, s a t}\left(\delta, M_{*}\right)-f_{r, c e n}\left(M_{*}\right)}{f_{b, c e n}\left(M_{*}\right)}
$$

in which $f_{b, c e n}\left(M_{*}\right)$ is the blue fraction of centrals. Within the hierarchical scenario, all satellites were centrals in the past, and the $\epsilon_{s a t}\left(\delta, M_{*}\right)$ quantity corresponds to the excess fraction of satellites which are environmentally quenched after the infall into a larger halo with respect to the population of star-forming centrals of the same mass.

The $\epsilon_{\text {sat }}\left(\delta, M_{*}\right)$ values computed from the zCOSMOS data are shown in the right-hand panels in Fig. 2 as squares. Given the broadness of our $\delta$-bins we also measure $\epsilon_{\text {sat }}\left(\delta, M_{*}\right)$ 

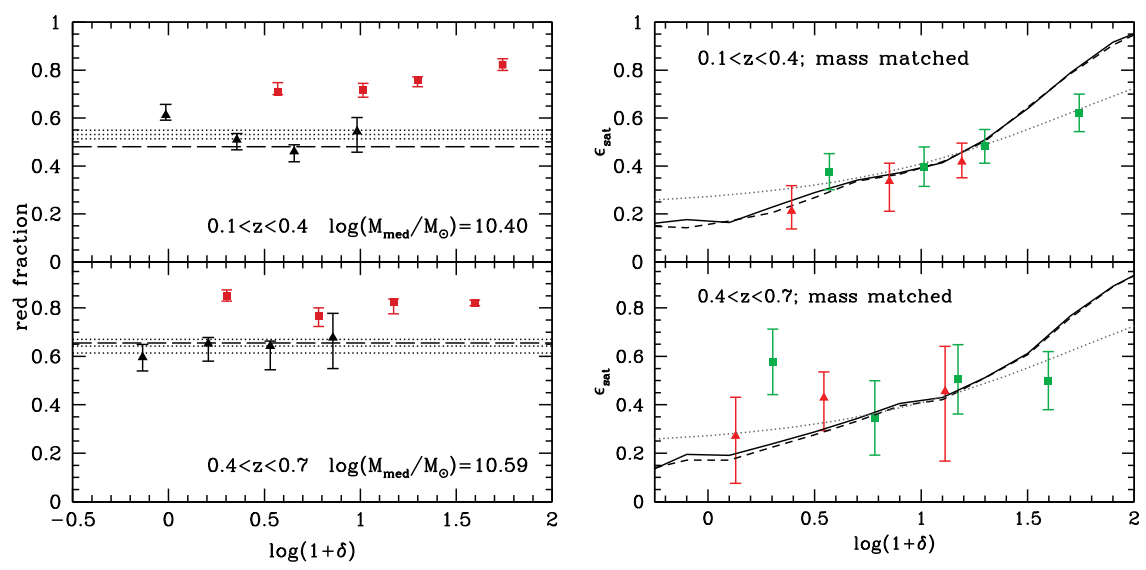

Figure 2. Left: Red fractions of centrals (triangles) and satellites (squares), matched in stellar mass, as a function of overdensity. The fractions measured in $0.1<z<0.4$ and $0.4<z<0.7$ are shown in the top and bottom panels, respectively. The errors encompass the $16-84 \%$ interval of the red fractions in the 20 realisations of the mass-matching. The dotted lines mark the average $f_{r, c e n}$ and its $\pm 1 \sigma$ values. The dashed lines mark the model $f_{r, c e n}$ values produced through the mass quenching alone. Right: Satellite quenching efficiency $\epsilon_{s a t}$ as a function of overdensity. The squared and triangular symbols correspond to the measurements in the $\delta$-quartiles and in the narrower bins of overdensity, respectively. The error bars encompass the $16-84 \%$ uncertainty interval based on the 20 (squares) or 50 (triangles) realisations of the mass-matching. The continuous and dashed lines are the model predicted $\epsilon_{\rho}(\delta) / f_{\text {sat }}\left(\delta, M_{*}\right)$ functions when using the satellite fraction measured in the $\Delta \log \left(M_{*} / M_{\odot}\right)=0.5$ bin centered at $\log \left(M_{*} / M_{\odot}\right)=10.40$ (top) and 10.59 (bottom), and 10.24 (top) and 10.49 (bottom), respectively. The dotted line is the equivalent $z \sim 0 \epsilon_{s a t}(\delta)$ measurement from Peng et al. (2012). Figures adapted from Kovač et al. (2014).

in a narrower range of overdensities where both populations are numerous, still matching the mass distribution of centrals to the satellites in a given $\delta$-bin. These $\epsilon_{\text {sat }}\left(\delta, M_{*}\right)$ estimates are shown as triangles in the right-hand panels in Fig. 2. The median stellar mass, when going from the lower to the higher narrower overdensity bins, takes values $\log \left(M_{*} / M_{\odot}\right)=10.24,10.32$ and 10.38 and $\log \left(M_{*} / M_{\odot}\right)=10.49,10.52$, and 10.60 in $0.1<z<0.4$ and $0.4<z<0.7$, respectively.

Within our mass and environment quenching model, in the situation when the quenched fraction of centrals is produced via the mass quenching process $\epsilon_{m}$ alone, the overall environment quenching $\epsilon_{\rho}$ must come from the satellite population. Then, for the fraction of satellites described by $f_{\text {sat }}\left(\delta, M_{*}\right)$, the equality between $\epsilon_{\text {sat }}\left(\delta, M_{*}\right)$ and $\epsilon_{\rho}(\delta) / f_{\text {sat }}\left(\delta, M_{*}\right)$ must hold.

The $\epsilon_{\rho}(\delta) / f_{\text {sat }}\left(\delta, M_{*}\right)$ functions obtained from the zCOSMOS data are shown as the black continuous and dashed lines in the right-hand panels in Fig. 2. Different curves are for the fraction of satellites measured at the median stellar mass in the reference overdensity bin and in the lowest median stellar mass in the narrower overdensity bins.

There is an excellent agreement between the purity-corrected $\epsilon_{s a t}\left(\delta, M_{*}\right)$ measurements in the narrower $\delta$-bins and $\epsilon_{\rho}(\delta) / f_{\text {sat }}\left(\delta, M_{*}\right)$ in both redshifts. The $\epsilon_{\text {sat }}\left(\delta, M_{*}\right)$ values from the quartiles in the overdensity are somewhat discrepant with the prediction, particularly in the lowest and highest overdensities. However, these points cover the broadest range in overdensity and the satellite red fractions measured in these intervals are rather smoothed. Considering the associated uncertainties, the agreement between the measured $\epsilon_{\text {sat }}\left(\delta, M_{*}\right)$ values and the $\epsilon_{\rho}(\delta) / f_{\text {sat }}\left(\delta, M_{*}\right)$ function is remarkably good. 
Our results constrain that the satellite galaxies must be the dominant population of galaxies driving the overall environmental trends at least up to $z<0.7$.

\section{Conclusions}

We have explored the colour-density relation in the final sample of galaxies in $0.1<$ $z<0.7$ obtained in the zCOSMOS-bright survey. Using the two environmental indicators: local overdensity of galaxies and the dichotomous sample of centrals and satellites, we reach the following conclusions: 1) red fraction of galaxies $f_{r e d}$ increases with both stellar mass and overdensity in $0.1<z<0.7$. Within the errors, $f_{\text {red }}$ is consistent to be separable in stellar mass and environment, indicating the existence of the two independent quenching mechanisms: mass quenching and environment quenching; 2) the differential effect of these two mechanisms does not change with cosmic time and they seem to be the same at $z=0.7$ as locally; 3 ) red fraction of centrals is consistent with being independent of overdensity and more than $95 \%$ of $f_{r, c e n}$ can be produced through the mass quenching alone; 4) the red fraction of satellites requires an additional quenching mechanism with respect to the mass quenching: at the same stellar mass and overdensity, satellites are redder; 5) in the framework of the model of mass and environment quenching efficiencies, our data support the scenario where the satellite quenching efficiency can explain the majority of the overall environmental effects at least up to $\mathrm{z}=0.7 ; 6$ ) given the associated uncertainties in our analysis, all these statements should be understood only as approximations to a complex physical reality. In our ongoing work, we are focussing on furthering the understanding of the physical processes governing the observed relations.

\section{References}

Baldry, I. K., Balogh, M. L., Bower, R. G., Glazebrook, K., Nichol, R. C., Bamford, S. P., \& Budavari, T., 2006, MNRAS, 373, 469

Boselli, A. \& Gavazzi, G., 2006, PASP, 118, 517

Capak, P. et al., 2007, ApJS, 172, 99

Cooper, M. C. et al., 2010, MNRAS, 409, 337

Cucciati, O. et al., 2010, A\&\&A, 524, A2

Dressler, A., 1980, ApJ, 236, 351

Henriques, B. M. B., White, S. D. M., Lemson, G., Thomas, P. A., Guo, Q., Marleau, G.-D., \& Overzier, R. A., 2012, MNRAS, 421, 2904

Kauffmann, G., White, S. D. M., Heckman, T. M., Ménard B., Brinchmann, J., Charlot, S., Tremonti, C., \& Brinkmann, J., 2004, MNRAS, 353, 713

Knobel, C. et al., 2012, ApJ, 753, 121

Knobel, C. et al., 2013, ApJ, 769, 24

Knobel, C., Lilly, S. J., Woo, J., \& Kovač K., 2014, arXiv1408.2553

Kovač K. et al., 2010b, ApJ, 708, 505

Kovač K. et al., 2010a, ApJ, 718, 86

Kovač K. et al., 2014, MNRAS, 438, 717

Lilly, S. J. et al., 2007, ApJS, 172, 70

Lilly, S. J. et al., 2009, ApJS, 184, 218

Peng, Y.-j. et al., 2010, ApJ, 721, 193

Peng, Y.-j., Lilly, S. J., Renzini, A., \& Carollo, M., 2012, ApJ, 757, 4

Sanders, D. B. et al., 2007, ApJS, 172, 86

Scoville, N. et al., 2007, ApJS, 172, 38

Tasca, L. A. M. et al., 2009, A\&A, 503, 379

van den Bosch F. C., Aquino, D., Yang, X., Mo, H. J., Pasquali, A., McIntosh, D. H., Weinmann, S. M., \& Kang, X., 2008, MNRAS, 387, 79 